\title{
Conditioned jaw movement in the rabbit: Deprivation procedure and saccharin concentration'
}

\author{
S. R. COLEMAN, M. M. PATTERSON AND I. GORMEZANO \\ UNIVERSITY OF IOWA
}

A new deprivation procedure produced an asymptotic increase over a previous study of $10-13 \%$ conditioned jaw movement. Effects of saccharin were not quite significant, but in the expected direction. In acquisition nonreinforced trials reduced response probability on the succeeding trial by $5-10 \%$, with no changes over days or groups.

Smith, DiLollo, \& Gormezano (1966) demonstrated classical appetitive conditioning of jaw movement elicited by a squirt of $.004 \%$ saccharin solution to the oral cavity of the rabbit. However, the paired CS-US group after eight sessions of 40 trials each reached an asymptotic performance level of only $72 \%$. Furthermore the procedure involved conditioning sessions on alternate days, imposed by a 48-hr. water deprivation schectule. The purpose of the present two experiments was respectively: (1) to develop a deprivation procedure which would produce higher asymptotic performance, without the use of alternate days, and (2) to vary the saccharin concentration of the US to determine its effect on conditioning.

Method

The Ss were 75 naive albino rabbits, 100 days old, housed individually, with free access to food. Six Ss at a time were run in two ledger-size fireproof threedrawer ventilated file cabinets by being placed in a restraining box and positioned 4 in. in front of a speaker mounted on a panel at the front of each drawer. On S's muzzle-like head mount was located a jaw-movement recording device consisting of a photocell and bulb, separated by a metal flag mounted on a horizontal shaft. Angled piano wire attached to this shaft was inserted into the channel of a wound clip permanently applied to the tip of S's lower jaw, and held firmly up against the jaw by a small spring. Jaw movements rotated the flag and the photocell signal was amplified and recorded. From a separate water reservoir 51 in. above the base of each drawer $3 / 16$ in. tubing, interrupted by a solenoid valve, passed down to the head mount and mated with $1 / 8 \mathrm{in}$. tubing that terminated in a blunted No. 16 hypodermic needle inserted into a polyethylene plug in S's left cheek. The CS was a $72 \mathrm{db}$, 1000-cps tone of 500 msec. duration and the US a $1.2 \mathrm{cc}$ squirt of water (or saccharin) to the oral cavity. The duration of the US was $300 \mathrm{msec}$. and occurred at the termination of the CS. In acquisition a deflection of the recording pen $1 \mathrm{~mm}$ or more in the interval from CS onset to $520 \mathrm{msec}$. after CS onset was scored as a CR.
In extinction the scoring interval was extended to 800 msec.

Seven days prior to the first training session $\mathrm{S}$ was anesthetized, the left cheek depilated, and a .085 in. diameter hole punched in the cheek. A PE 205 polyethylene tube was inserted, flared at both ends, held in place by two polyethylene washers, and a wound clip applied to the tip of S's lower jaw. Two days of recovery followed with free access to water in the home cage. Then for five successive days $S$ was given $3 \mathrm{~min}$. access to water. On the fifth day $S$ was habituated to the restraining box, and on the following day acquisition was begun. All groups in the two experiments received 10 days of acquisition and two days of extinction of 50 trials per day at an average intertrial interval of 90 sec. Trials $5,15,25,35$, and 45 of each acquisition session were nonreinforced. In Experiment 1, 12 Ss were randomly assigned to each of two groups. For Group W-Sup, $3 \mathrm{~min}$. access to water following each acquisition session was maintained throughout acquisition, whereas for Group $W$ access was discontinued. Both groups were given $3 \mathrm{~min}$. access to water after an extinction session. For Experiment 2, 17 Ss were randomly assigned to each of three groups differing in saccharin concentration of the US, of $.260 \%, .087 \%$, and $.029 \%$ for Groups 260,87 , and 29 respectively. For all groups, the $3 \mathrm{~min}$. access to water was terminated at the beginning of acquisition and reinstated in extinction. Results

Figures 1 and 2 present acquisition and extinction functions for Experiments 1 and 2 respectively, plotted over blocks of nine reinforced trials within each acquisition day and blocks of 10 trials in extinction. In Fig. 1 response probability is shown to increase from about $20 \%$ on Day 1 to a terminal level of about $85 \%$ on Day 10 . Daily within-session decrements of about 10-20\% were observed. Blocks and Days effects were highly significant (both p's < .001). Although Group $W$ was responding at a somewhat higher terminal acquisition level, Groups W and W-Sup did not reliably differ. Within-session decrements were largest on Days 3 to 6 , and this was reflected in a significant Days by Blocks effect $(F=1.47, d f=36 / 792, p=.05)$. Examination of Fig. 2 reveals that the performance differences of Groups 260,87 , and 29 were in the expected direction, with Group 260 showing the most rapid conditioning and highest asymptotic response level of about $90 \%$. Group differences, however, were not quite significant ( $F=$ 

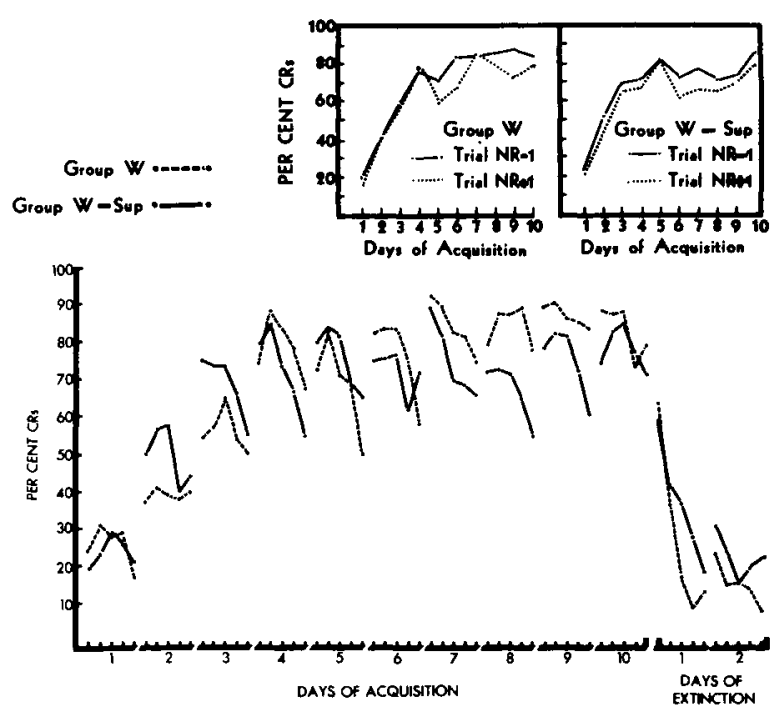

Fig. 1. Percentage CRs in acquisition and extinction. Insets present in 5 -trial blocks response probabilities for the trial immediately following (Trial $\mathrm{NR}+1$ ) and preceding (Trial NR-1) a nonreinforced trial.

2.77, $d f=2 / 48, .05<p<.10$ ). No substantial differences in magnitude of within-session decrements appeared among groups as revealed by a nonsignificant Blocks by Groups interaction. Blocks and Days effects were highly significant (both $\mathrm{p}$ 's $<.001$ ) and again the significant Blocks by Days interaction $(F=1.75$, $\mathrm{df}=$ $36 / 1728, p<.05$ ) reflected larger within-session decrements on Days 3 to 6 . In the extinction data of Fig. 1 and 2, all groups demonstrated a gradual performance decrease to terminal levels of about $20 \%$ on Days 1 and 2. Separate analyses of variance for each experiment across extinction days revealed a significant Blocks
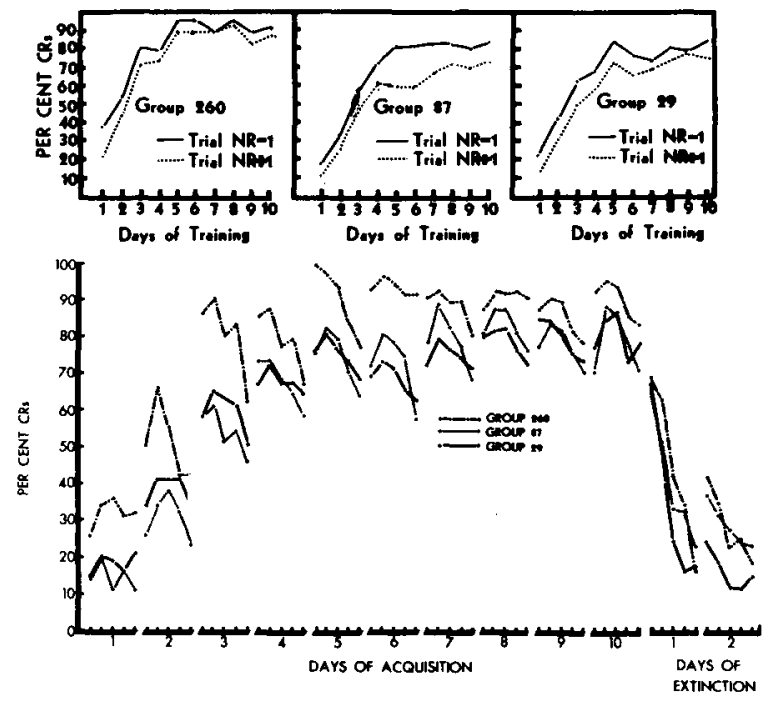

Fig. 2. Percentage CRs in acquisition and extinction. Insets present in 5-trial blocks response probabilities for the trial immediately following (Trial NR+1) and preceding (Trial NR-1) a nonreinforced trial. effect ( $p<.001$ in both studies) but no significant main or interaction effects of Groups. Analyses of variance were performed on the response probabilities over the last 10 trials of Day 1 of extinction and the first 10 trials of Day 2. These analyses revealed a significant amount of spontaneous recovery ( $p<.05$ in both studies), but again no significant main or interaction effects of Groups.

The insets in Fig. 1 and 2 indicate that the response probabilities on the trial immediately following a nonreinforced trial (Trial $\mathrm{NR}+1$ ) are consistently lower across Days and Groups than probabilities on the trial immediately preceding a nonreinforced trial (Trial NR-1). A nonsignificant Trial by Blocks interaction reflected the fact that the Trial NR+1 and Trial NR-1 curves were virtually parallel over days, and a nonsignificant interaction with Groups indicated a virtual constancy of the curve differences over groups.

\section{Discussion}

An altered deprivation procedure indicated an asymptotic increase of $10-13 \%$ over the Smith et al (1966) study, and the data suggest that the absence of supplementary watering (Group W) leads to higher asymptotic responding than does its maintenance (Group W-Sup). Furthermore, effects of saccharin concentration of the US were in the expected direction, even though only a limited range of concentrations were explored. Lastly, as with Smith et al, within-session decrements were still observed.

Compared to classical conditioning studies on other organisms, the extinction performance was gradual, but compared to other preparations with the rabbit, i.e., external eyelid, nictitating membrane, and retractor bulbi, the extinction process was relatively rapid. In acquisition, nonreinforced trials caused large response decrements on the following trial, and these decrements were surprisingly constant over days. This constancy of decremental effects of nonreinforcement in acquisition contrasts sharply with the observation that partially reinforced $(50 \%)$ groups in the nictitating membrane preparation reach the same asymptotic response level of $95-100 \%$ as do groups on continuous reinforcement (Coleman et al, 1966). The relatively rapid extinction and the failure of decremental effects of nonreinforced trials in acquisition to disappear with continued training indicates the sensitivity of the present preparation to effects of nonreinforcement, in contrast to the robustness of the membrane preparation.

\section{References}

Coleman, S. R., Patterson, M. M., \& Gormezano, I. Partial reinforcement and intertrial interval: Effect on classical conditioning and extinction of the.rabbit nictitating membrane. Midwestern Psychological Association, Chicago, 1966.

Smith, M. C., DiLollo, V., \& Gormezano, I. Conditioned jaw movement in the rabbit. J. comp. physiol. Psychol., 1966, in press.

\section{Note}

1. This research was supported by NSF Grant GB-2843 at Indiana University and a NSF Predoctoral Fellowship to $S$. R. Coleman. 